\title{
Locating and Identifying Codes in Circulant Graphs
}

\author{
Shu Jiao Song $\mathbb{D}$, Weiqian Zhang, and Can Xu \\ Yantai University, Yantai, China \\ Correspondence should be addressed to Shu Jiao Song; shujiao.song@hotmail.com
}

Received 31 August 2021; Accepted 29 September 2021; Published 28 October 2021

Academic Editor: Akbar Ali

Copyright (C) 2021 Shu Jiao Song et al. This is an open access article distributed under the Creative Commons Attribution License, which permits unrestricted use, distribution, and reproduction in any medium, provided the original work is properly cited.

Identifying and locating-dominating codes have been studied widely in circulant graphs. Recently, Ville Junnila et al. (Optimal bounds on codes for location in circulant graphs, Cryptography and Communications; 2019) studied identifying and locatingdominating codes in circulants $C_{n}(1, d), C_{n}(1, d-1, d)$, and $C_{n}(1, d-1, d, d+1)$. In this paper, identifying, locating, and selfidentifying codes in the circulant graphs $C_{n}(k, d), C_{n}(k, d-k, d)$, and $C_{n}(k, d-k, d, d+k)$ are studied, and this extends Junnila et al.'s results to general cases.

\section{Introduction}

Let $\Gamma=(V, E)$ be a graph with vertex set $V$ and edge set $E$. We say vertices $u$ and $v$ are adjacent, if there is an edge between $u$ and $v$ in $\Gamma$. The distance $d(u, v)$ is the number of edges of a shortest path between the vertices $u$ and $v$, and it is denoted by $d(u, v)$. For any vertex $u \in V$, the open neighborhood of $u$ in $\Gamma$ is denoted by $N_{\Gamma}(u)=\{v \in V \mid d(u, v)=1\}$. The closed neighborhood of $u \in V$ in $\Gamma$ is denoted by $N_{\Gamma}[u]=N_{\Gamma}(u) \cup\{u\}$.

Given a group $G$ and a subset $S$ of $G$, the graph $\Gamma=$ $\operatorname{Cay}(G, S)$ is a Cayley graph on $G$ with respect to $S$. The vertices in $\Gamma$ are the elements of $G$ and vertices $x$ and $y$ are adjacent if and only if $y x^{-1} \in S$. It is known that Cay $(G, S)$ is connected if and only if $S$ generates $G$. If $G$ is a cyclic group, then $\Gamma$ is called a circulant graph. We denote Cay $\left(\mathbb{Z}_{n}, S\right)$, $S=\left\{d_{1}, d_{2}, \ldots, d_{k}\right\}$, by $C_{n}\left(d_{1}, d_{2}, \ldots, d_{k}\right)$, where $n$ and $d_{i}$ are positive integers such that $d_{i} \leq n / 2$ for all $i=1,2, \ldots, k$. The vertex set of the circulant graph is $V=\mathbb{Z}_{n}=\{0,1,2, \ldots, n-1\}$, and the open neighborhood of arbitrary vertex $u \in V$ is $N(u)=\left\{u-d_{1}, u-\right.$ $\left.d_{2}, \ldots, u-d_{k}\right\} \cup\left\{u+d_{1}, u+d_{2}, \ldots, u+d_{k}\right\}$.

A nonempty subset of vertices $C \subseteq V$ is called code and its elements are called codewords, and the number of elements in it is called the order of $C$ denoted by $|C|$. For a finite nonempty set $S \subseteq V$ in a graph $\Gamma=(V, E)$, we define the density of a code $C \subseteq V$ in $S$ by $|S \cap C| /|S|$. A code $C$ is called dominating if every vertex $u$ of $\Gamma$ is either in $C$ or it has a neighbor in $C$. The $I$-set of a vertex $u$ depending on a code $C$ is defined by

$$
I(G, C ; u)=N[u] \cap C .
$$

A set $C \subseteq V(\Gamma)$ is called a locating code in $\Gamma$, if $C$ is a dominating code and for any distinct vertices $u, v \in V, C$, we have $I(C ; u) \neq I(C ; v)$. In a finite graph $\Gamma$, the smallest possible order of a locating code is denoted by $\gamma^{\mathrm{LD}}(\Gamma)$. A locating-dominating code with order $\gamma^{\mathrm{LD}}(\Gamma)$ is called $o p$ timal. A set $C \subseteq V(\Gamma)$ is called an identifying code in $\Gamma$, if $C$ is a dominating code and for any distinct vertices $u, v \in V$, we have $I(C ; u) \neq I(C ; v)$. The smallest possible order of an identifying code in a finite graph $\Gamma$ is denoted by $\gamma^{\mathrm{ID}}(\Gamma)$. An identifying code with $\gamma^{\mathrm{ID}}(\Gamma)$ codewords is called optimal. A code $C \subseteq V$ is self-identifying in $\Gamma=(V, E)$ if for all distinct $u, v \in V, I(C ; u), I(C ; v) \neq \varnothing$. In addition, there is an equivalent definition of self-identifying code $[1,2]: C$ is a self-identifying code in $\Gamma$ if and only if for all $u \in V$, we have $I(C ; u) \neq \varnothing$ and $\cap_{c \in I(C ; u)} N[c]=\{u\}$. In a finite graph $\Gamma$, the smallest possible order of a self-identifying code is denoted by $\gamma^{\operatorname{SID}}(\Gamma)$ and a self-identifying code with order $\gamma^{\operatorname{SID}}(\Gamma)$ is called optimal.

By definition, it is easy to see every identifying code is also a locating code and a self-identifying code is also an identifying code. 
In 1988 , to promote nuclear power plant safety, locating codes were first introduced by P. J. Slater [3]; vertices of a locating-dominating set $S$ correspond to safeguards and an intruder corresponding to a vertex in $V, S$. In 1998, identifying codes were first introduced in a more general form by Karpovsky et al. [4].

Locating and identifying codes of some special graphs have been studied (see [5-10] for reference). Great efforts are made in studying locating and identifying codes in circulants. M. Ghebleh and L. Niepel studied locating and identifying code numbers of $C_{n}(1,3)$ in $[11]$, but they stated as an open question what happens in the graphs $C_{n}(1, d)$ with $d$ being greater than 3 and mentioned that the methods used in their paper seem to be nonapplicable. So, Ville Junnila, Tero Laihonen, and Gabrielle Paris present a new approach to study identifying, locating-dominating, and self-identifying codes in the circulant graphs $C_{n}(1, d), C_{n}(1, d-1, d)$, and $C_{n}(1, d-$ $1, d, d+1)$ using suitable grids in [12]. However, there is no relevant conclusion for the more general 4,6,8 degree circulant graphs. This is the motivation of this paper.

Theorem 1. Let $n, k$, and $d$ be positive integers such that $n>2 d>2 k \geq 2,(k, d, n)=1$, and $\Gamma=C_{n}(k, d)$. Then, $\Gamma$ is connected and

$$
\begin{gathered}
\gamma^{\mathrm{ID}}(\Gamma) \geq\left\lceil\frac{7 n}{20}\right\rceil, \\
\gamma^{\mathrm{LD}}(\Gamma) \geq\left\lceil\frac{3 n}{10}\right\rceil, \\
\gamma^{\mathrm{SID}}(\Gamma) \geq\left\lceil\frac{n}{2}\right\rceil .
\end{gathered}
$$

Theorem 2. Let $n, k$, and $d$ be positive integers such that $n>2 d>4 k,(k, d, n)=1$, and $\Gamma=C_{n}(k, d-k, d)$. Then, $\Gamma$ is connected and

$$
\begin{gathered}
\gamma^{\mathrm{ID}}(\Gamma) \geq\left\lceil\frac{n}{4}\right\rceil, \\
\gamma^{\mathrm{SID}}(\Gamma) \geq\left\lceil\frac{n}{2}\right\rceil .
\end{gathered}
$$

Theorem 3. Let $n, k$, and $d$ be positive integers such that $n>2 d>4 k, \quad 2 d+k \neq n, \quad 2 d+2 k \neq n, \quad(k, d, n)=1$, and $\Gamma=C_{n}(k, d-k, d, d+k)$. Then, $\Gamma$ is connected and

$$
\begin{aligned}
& \gamma^{\mathrm{LD}}(\Gamma) \geq\left\lceil\frac{n}{5}\right\rceil, \\
& \gamma^{\mathrm{ID}}(\Gamma) \geq\left\lceil\frac{2 n}{9}\right\rceil, \\
& \gamma^{\mathrm{SID}}(\Gamma) \geq\left\lceil\frac{n}{3}\right\rceil .
\end{aligned}
$$

\section{Remark 1}

(i) There is an infinite sequence of identifying codes in the circulant graphs $C_{n}(k, d-k, d)$ such that their density tends to $1 / 4$. (ii) We have an infinite sequence of identifying codes in the circulant graphs $C_{n}(k, d-k, d, d+k)$ such that their density tends to $2 / 9$.

(iii) All other bounds given above are optimal. Examples are given in this paper.

\section{Infinite Grids and Circulant Graphs}

In order to study the codes of circulant graphs, we first give the necessary and sufficient conditions of these circulants to be connected.

Lemma 1. Let $n$ and $d$ be positive integers and $d \geq 2$. Then, $C_{n}(k, d), C_{n}(k, d-k, d)$, and $C_{n}(k, d-k, d, d+k)$ are connected if and only if $(k, d, n)=1$.

Proof. Let $(d, n)=d_{1}$ and $(k, n)=k_{1}$. Then, there exist $x, y \in \mathbb{Z}$ such that $d x=d_{1}(\bmod n)$ and $k y=k_{1}(\bmod n)$. If $(k, d, n)=1$, then $\left(d_{1}, k_{1}, n\right)=1$. Hence, there exist $t, m \in \mathbb{Z}$ such that $d_{1} t+k_{1} m=s_{1}(\bmod n)$, where $\left(s_{1}, n\right)=1$. So, there exists $t_{0} \in \mathbb{Z}$ such that $s_{1} t_{0}=1(\bmod n)$, and then we have $d_{1} t t_{0}+k_{1} m t_{0}=1(\bmod n)$ and $d x t t_{0}+k y m t_{0}=1(\bmod n)$. So, $d a+k b=1(\bmod n)$, where $x t t_{0}=a$ and $y m t_{0}=b$. Thus, $\langle k, d\rangle=\mathbb{Z}_{n}, C_{n}(k, d), C_{n}(k, d-k, d)$, and $C_{n}(k, d-k, d, d+$ $k)$ are connected.

On the contrary, if $(k, d, n)=l>1$, we have $\langle k, d\rangle=\langle l\rangle\left\langle\mathbb{Z}_{n}\right.$, and $C_{n}(k, d)$ is not connected. In addition, $\langle k, d-k, d\rangle=\langle k, d-k, d, d+k\rangle=\langle k, d\rangle$.

Next, we give definitions of infinite square, triangular, and king grids.

(i) The square grid $\mathcal{S}$ : the vertex set of the square grid is $V=\mathbb{Z}^{2}$, and vertex $u=(x, y) \in \mathbb{Z}^{2}$ is adjacent to $v$ if and only if $v \in\{(x \pm 1, y),(x, y \pm 1)\}=N(\mathcal{S} ; u)$ (see Figure 1(a) for details).

(ii) The triangular grid $\mathscr{T}$ : the vertex set of the triangular grid is $V=\mathbb{Z}^{2}$, and vertex $u=(x, y) \in \mathbb{Z}^{2}$ is adjacent to $v$ if and only if $v \in N(\mathcal{\delta} ; u) \cup\{(x+1, y+$ $1),(x-1, y-1)\}=N(\mathscr{T} ; u)$ (see Figure $1(\mathrm{~b})$ for details).

(iii) The king grid $\mathscr{K}$ : the vertex set of the king grid is $V=\mathbb{Z}^{2}$, and vertex $u=(x, y) \in \mathbb{Z}^{2}$ is adjacent to $v$ if and only if $v \in N(\mathscr{T} ; u) \cup\{(x+1, y-1),(x-1, y+$ $1)\}=N(\mathscr{K} ; u)$ (see Figure 2 for details).

In order to study the codes of circulant graphs, we give correspondences between circulant graphs and grids.

(i) Let $\Gamma=C_{n}(k, d)$ be a circulant graph. Let $\varphi_{1}$ be the mapping from the vertices in the square grid to vertices in $\Gamma$, such that

$$
\varphi_{1}: x=(i, j) \longmapsto i \cdot k+j \cdot d(\bmod n) .
$$

(ii) Let $\Gamma=C_{n}(k, d-k, d)$ be a circulant graph. Let $\varphi_{2}$ be the mapping from the vertices in the triangular grid to vertices in $\Gamma$, such that 


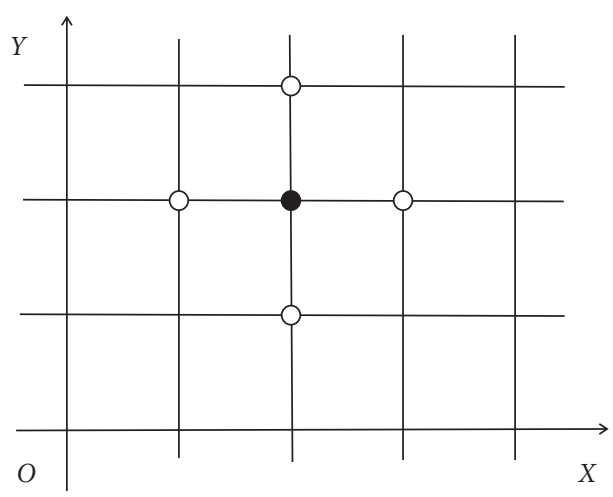

(a)

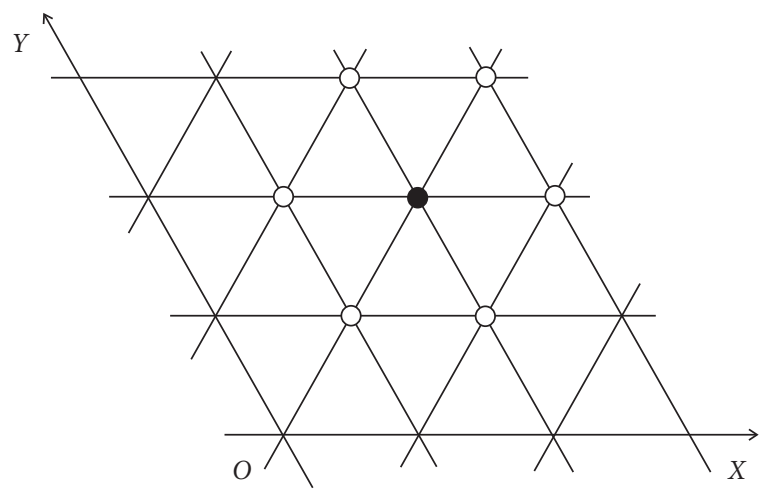

(b)

FIgURE 1: (a) The close neighborhood of $u$ in the square grid $\mathcal{S}$, and $u=(x, y) \in \mathbb{Z}^{2}$ is denoted by a round black spot. Its open neighborhood is the set of all the hollow dots. (b) The close neighborhood of $u$ in the triangular grid $\mathscr{T}$, and $u=(x, y) \in \mathbb{Z}^{2}$ is denoted by a black spot. Its open neighborhood is the set of all the hollow dots.

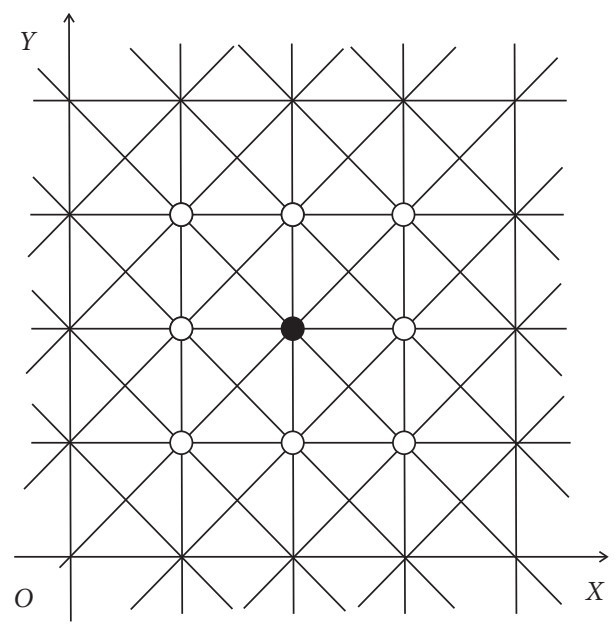

FIGURE 2: The close neighborhood of $u$ in the king grid $\mathcal{S}$, and $u=(x, y) \in \mathbb{Z}^{2}$ is denoted by a black spot. Its open neighborhood is the set of all the hollow dots.

$\varphi_{2}: x=(i, j) \longmapsto i \cdot k+j \cdot(d-k)(\bmod n)$.

(iii) Let $\Gamma=C_{n}(k, d-k, d, d+k)$ be a circulant graph. Let $\varphi_{3}$ be the mapping from the vertices in the square grid to vertices in $\Gamma$, such that $\varphi_{3}: x=(i, j) \longmapsto i \cdot k+j \cdot d(\bmod n)$.

Now, we will prove that these maps keep the adjacency relationships.

(i) By the definition of $\varphi_{1}$, vertex $(i, j)$ in the square grid $\delta$ corresponds to the vertex $i \cdot k+j \cdot d(\bmod n)$ in $C_{n}(k, d)$, then

$$
(i, j) \text { is adjacent to }\left\{\begin{array} { l } 
{ ( i , j + 1 ) } \\
{ ( i , j - 1 ) } \\
{ ( i + 1 , j ) } \\
{ ( i - 1 , j ) }
\end{array} \Longleftrightarrow i \cdot k + j \cdot d \text { is adjacent to } \left\{\begin{array}{l}
i \cdot k+(j+1) \cdot d, \\
i \cdot k+(j-1) \cdot d, \\
(i+1) \cdot k+j \cdot d, \\
(i-1) \cdot k+j \cdot d
\end{array}\right.\right.
$$

Therefore, the map keeps the adjacency relationships. 
(ii) By the definition of $\varphi_{2}$, vertex $(i, j)$ in the triangular grid $\mathscr{T}$ corresponds to the vertex $i \cdot k+j \cdot(d-k)(\bmod n)$ in $C_{n}(k, d-k, d)$, then

$$
(i, j) \text { is adjacent to }\left\{\begin{array} { l } 
{ ( i , j + 1 ) } \\
{ ( i , j - 1 ) } \\
{ ( i + 1 , j ) } \\
{ ( i - 1 , j ) } \\
{ ( i + 1 , j + 1 ) } \\
{ ( i - 1 , j - 1 ) }
\end{array} \Longleftrightarrow i \cdot k + j \cdot ( d - k ) \text { is adjacent to } \left\{\begin{array}{l}
i \cdot k+(j+1) \cdot(d-k), \\
i \cdot k+(j-1) \cdot(d-k), \\
(i+1) \cdot k+j \cdot(d-k), \\
(i-1) \cdot k+j \cdot(d-k), \\
(i+1) \cdot k+(j+1) \cdot(d-k), \\
(i-1) \cdot k+(j-1) \cdot(d-k) .
\end{array}\right.\right.
$$

Therefore, the map keeps the adjacency relationships.

(iii) By the definition of $\varphi_{3}$, vertex $(i, j)$ in the king grid $\mathscr{K}$ corresponds to the vertex $i \cdot k+j \cdot d(\bmod n)$ in $C_{n}(k, d-k, d, d+k)$, then

$$
(i, j) \text { is adjacent to }\left\{\begin{array} { l } 
{ ( i , j + 1 ) } \\
{ ( i , j - 1 ) } \\
{ ( i + 1 , j ) } \\
{ ( i - 1 , j ) } \\
{ ( i + 1 , j + 1 ) } \\
{ ( i - 1 , j - 1 ) } \\
{ ( i - 1 , j + 1 ) } \\
{ ( i + 1 , j - 1 ) }
\end{array} \Longleftrightarrow i \cdot k + j \cdot d \text { is adjacent to } \left\{\begin{array}{l}
i \cdot k+(j+1) \cdot d, \\
i \cdot k+(j-1) \cdot d, \\
(i+1) \cdot k+j \cdot d, \\
(i-1) \cdot k+j \cdot d, \\
(i+1) \cdot k+(j+1) \cdot d, \\
(i-1) \cdot k+(j-1) \cdot d, \\
(i-1) \cdot k+(j+1) \cdot d, \\
(i+1) \cdot k+(j-1) \cdot d .
\end{array}\right.\right.
$$

Therefore, the map keeps the adjacency relationships.

Next, we give the relationship about identifying, locating-dominating, and self-identifying code between the circulants $C_{n}(k, d), C_{n}(k, d-k, d)$, and $C_{n}(k, d-k, d, d+k)$ and the square, triangular, and king grids.

Lemma 2. Let $n, d, k$, and $t$ be positive integers such that $n>2 d>2 k \geq 2$. If $C$ is an identifying code in $C_{n}(k, d)$ with order $t$, then there is an identifying code in the infinite square grid $\mathcal{S}$ with the density $t / n$. Analogous conclusions remain true for locating and self-identifying codes.

Proof. Let $\Gamma=C_{n}(k, d)$ be a circulant graph, and $C$ an identifying code in $\Gamma$. Let $C_{\mathcal{S}}=\left\{(i, j) \in \mathbb{Z}^{2} \mid i \cdot k+j \cdot d \in C\right\}$ be a code in $\mathcal{S}$.

First, we show that $C_{\mathcal{S}}$ is an identifying code in the square grid $\delta$. Suppose there are two distinct vertices $x=$ $(i, j) \in \mathbb{Z}^{2}$ and $y=(i, j)+(a, b) \in \mathbb{Z}^{2}(a, b \in \mathbb{Z})$ in the square grid such that $I(\delta ; x)=I(\delta ; y)$. Because $C$ is a dominating set, so is $C_{\mathcal{S}}$, and then $I(\mathcal{S} ; x)$ and $I(\mathcal{S} ; y)$ are nonempty sets. So, we just need to consider the cases of $d_{\mathcal{S}}(x, y) \leq 2 \quad$ (if $d_{\mathcal{S}}(x, y) \geq 3$, then $N(x) \cap N(y)=\varnothing$,
$N(x) \neq N(y))$. Without less of generality, we assume $b \geq 0$ (otherwise, you just need to switch the roles of $x$ and $y$ ). So, $(a, b) \in S=\{(1,0),(-1,0),(0,1),(1,1),(-1,1)$,

$(2,0),(-2,0),(0,2)\}$. Because $I(\delta ; x)=I(\delta ; y)$, we have $I[\Gamma ; i \cdot k+j \cdot d]=I[\Gamma ;(i+a) \cdot k+(j+b) \cdot d]$. Moreover, because $C$ is an identifying code, we have $i \cdot k+j \cdot d \equiv(i+$ $a) \cdot k+(j+b) \cdot d(\bmod n)$. Hence, $a \cdot k+b \cdot d \equiv 0(\bmod n)$. However, $(a, b) \in S, I(\mathcal{S} ; x) \neq I(\mathcal{S} ; y)$ by verifying all cases of $(a, b)$ which contradicts to $I(\mathcal{S} ; x)=I(\mathcal{S} ; y)$, so $C_{\mathcal{\delta}}$ is an identifying code in $\mathcal{S}$.

The proof is similar for the locating codes. Just notice that the vertices $x=(i, j) \in \mathbb{Z}^{2}$ and $y=x+(a, b) \in \mathbb{Z}^{2}$ are non-codewords, and their corresponding vertices $i \cdot k+j \cdot d$ and $(i+a) \cdot k+(j+b) \cdot d$ are also non-codewords in $\Gamma$.

Now, we give the proof for self-identifying codes. By the definitions of identifying and self-identifying codes, we know every self-identifying code is also an identifying code. Suppose that $C$ is a self-identifying code in $\Gamma, C$ is also an identifying code in it. According to the above proof of identifying codes, we know $C_{\mathcal{S}}$ is an identifying code in $\mathcal{S}$. Next, we show that $I(\mathcal{S} ; x), I(\mathcal{S} ; y) \neq \varnothing$ for any distinct vertices $x=(i, j) \in \mathbb{Z}^{2}$ and $y=x+(p, q) \in \mathbb{Z}^{2}(p, q \in \mathbb{Z})$. 
Since $C_{\delta}$ is an identifying code, the result is true if $d_{\delta}(x, y) \geq 3$.

Suppose that $1 \leq d_{\delta}(x, y) \leq 2$, namely, $\quad(p, q) \in$ $\{(-1,-1),(0,-2),(1,-1),(2,0),(1,1), \quad(0,2),(-1,1)$, $(-2,0),(-1,0),(1,0),(0,1),(0,-1)\}$. Hence, the set $I(\Gamma ; i$. $k+j \cdot d), I(\Gamma ;(i+p) \cdot k+(j+q) \cdot d)$ contains a codeword of $C$ because $C$ is a self-identifying code in $\Gamma$. Notice that $i \cdot k+j \cdot d$ and $(i+p) \cdot k+(j+q) \cdot d$ are distinct vertices by verifying all of the above $(p, q)$ cases in $\Gamma$ as $n>2 d>2 k$. Thus, $I[\delta ; x], I[\delta ; y]$ always contains a codeword of $C_{\delta}$. This completes the proof.

Lemma 3. Let $n, d, k$, and $t$ be positive integers such that $n>2 d>4 k$. If $C$ is an identifying (locating or self $-i$ de ntifying) code in $C_{n}(k, d-k, d)$ with order $t$, then there is an identifying (locating or self identifying) code in the infinite triangular grid $\mathscr{T}$ with the density $t / n$.

Proof. Let $\Gamma=C_{n}(k, d-k, d)$ be a circulant graph and $C$ an identifying code in $\Gamma$. Let $C_{\mathscr{T}}=\left\{(i, j) \in \mathbb{Z}^{2} \mid i \cdot k+j \cdot(d-\right.$ $k) \in C$ be a code in $\mathscr{T}$.

Now, we claim that $C_{\mathscr{T}}$ is an identifying code in the triangular grid $\mathscr{T}$. Suppose that $C_{\mathscr{T}}$ is not an identifying code, so there are two distinct vertices $x=(i, j) \in \mathbb{Z}^{2}$ and $y=x+(a, b) \in \mathbb{Z}^{2}(a, b \in \mathbb{Z})$ such that $I(\mathscr{T}, x)=I(\mathscr{T}, y)$ in the triangular grid $\mathscr{T}$.

Because $C$ is a dominating set, so is $C_{\mathscr{T}}$, and then $I(\mathscr{T} ; x)$ and $I(\mathscr{T} ; y)$ are nonempty sets. So, we just need to consider the cases of $d_{\mathscr{T}}(x, y) \leq 2$ (if $d_{\mathscr{T}}(x, y) \geq 3$, then $N(x) \cap N(y)=\varnothing, N(x) \neq N(y))$. Without less of generality, we assume $b \geq 0$ (otherwise, you just need to switch the roles of $x$ and $y)$. So, $(a, b) \in T=\{(1,0),(-1,0),(1,1)$, $(-1,1),(2,0), \quad(-2,0),(0,2),(2,1),(1,2), \quad(2,2), \quad(0,1)\}$. Because $I(\mathscr{T} ; x)=I(\mathscr{T} ; y)$, we have $I(\Gamma ; i \cdot k+j \cdot(d-k))$ $=I(\Gamma ;(i+a) \cdot k+(j+b) \cdot(d-k))$. Moreover, because $C$ is an identifying code, we have $i \cdot k+j \cdot(d-k) \equiv(i+a)$ $\cdot k+(j+b) \cdot(d-k)(\bmod n)$. Hence, $a \cdot k+b \cdot d-b \cdot k \equiv 0$ $(\bmod n)$. However, $(a, b) \in T, I(\mathscr{T}, x) \neq I(\mathscr{T}, y)$ by verifying all cases of $(a, b)$ which contradicts to $I(\mathscr{T} ; x)$ $=I(\mathscr{T} ; y)$, so $C_{\mathscr{T}}$ is an identifying code in $\mathscr{T}$.

The proof is similar for the locating-dominating codes. Just notice that the vertices $x=(i, j) \in \mathbb{Z}^{2}$ and $y=x+(a, b) \in \mathbb{Z}^{2}$ are non-codewords in $\mathscr{T}$, and their corresponding vertices $i \cdot k+j \cdot(d-k)$ and $(i+a) \cdot k+(j+$ $b) \cdot(d-k)$ are also non-codewords in $\Gamma$.

Now, we give the proof for self-identifying codes. Suppose that $C$ is a self-identifying code in $\Gamma$ (it is also an identifying code) and $C_{\mathscr{T}}$ is an identifying code in $\mathscr{T}$. Next, we show that $I(\mathscr{T} ; x), I(\mathscr{T} ; y) \neq \varnothing$ for any distinct vertices $x=(i, j) \in \mathbb{Z}^{2}$ and $y=x+(p, q) \in \mathbb{Z}^{2}(p, q \in \mathbb{Z})$. Since $C_{\mathscr{T}}$ is an identifying code, the result is true if $d_{\mathscr{T}}(x, y) \geq 3$.

Suppose that $1 \leq d_{\mathscr{T}}(x, y) \leq 2$, namely, $(p, q) \in\{(-2$, $-1),(-2,-2),(-1,-2),(0,-2),(1,-1),(2,0),(2,1),(2,2)$, $(1,2),(0,2),(-1,1),(-2,0),(-1,0),(-1,-1),(0,-1),(1,0)$, $(1,1),(0,1)\}$.

Hence, the set $I(\Gamma ; i \cdot k+j \cdot(d-k)), I(\Gamma ;(i+p) \cdot k+$ $(j+q) \cdot(d-k))$ contains a codeword of $C$ because $C$ is a self-identifying code in $\Gamma$. Notice that $i \cdot k+j \cdot(d-k)$ and $(i+p) \cdot k+(j+q) \cdot(d-k)$ are distinct vertices by verifying all of the above $(p, q)$ cases in $\Gamma$ as $n>2 d>4 k$. Thus, $I[\mathscr{T} ; x], I[\mathscr{T} ; y]$ always contains a codeword of $C_{\mathscr{T}}$. We are done.

Lemma 4. Let $n, d, k$, and $t$ be positive integers such that $n>2 d>4 k, 2 d+k \neq n$, and $2 d+2 k \neq n$. If $C$ is an identifying code in $C_{n}(k, d-k, d, d+k)$ with order $t$, then there is an identifying code in the infinite king grid $\mathscr{K}$ with the density $t / n$. Analogous conclusions remain true for locating and selfidentifying codes.

Proof. Let $\Gamma=C_{n}(k, d-k, d, d+k)$ be a circulant graph and $C$ an identifying code in $\Gamma$. Let $C_{\mathscr{K}}=\left\{(i, j) \in \mathbb{Z}^{2} \mid i \cdot k+j \cdot d \in C\right\}$ be a code in $\mathscr{K}$.

We first show that $C_{\mathscr{K}}$ is an identifying code in the king grid $\mathscr{K}$. Suppose there are two distinct vertices $x=(i, j) \in \mathbb{Z}^{2}$ and $y=(i, j)+(a, b) \in \mathbb{Z}^{2}(a, b \in \mathbb{Z})$ in the king grid such that $I(\mathscr{K} ; x)=I(\mathscr{K} ; y)$. Because $C$ is a dominating set, so is $C_{\mathscr{K}}$, and then $I(\mathscr{K} ; x)$ and $I(\mathscr{K} ; y)$ are nonempty sets. So, we just need to consider the cases of $d_{\mathscr{K}}(x, y) \leq 2$ (if $d_{\mathscr{K}}(x, y) \geq 3$, then $N(x) \cap N(y)=\varnothing$ and $N(x) \neq N(y))$. Without loss of generality, we assume $b \geq 0$ (otherwise, you just need to switch the roles of $x$ and $y$ ). So, $(a, b) \in K=\{(0,1),(1,0),(1,1),(-1,1), \quad(-1,0),(0,2)$, $(2,0),(-2,0),(1,2),(-1,2),(2,2),(-2,2),(2,1),(-2,1)\}$. Because $I(\mathcal{S} ; x)=I(\mathcal{S} ; y)$, we have $I[\Gamma ; i \cdot k+j \cdot d]=$ $I[\Gamma ;(i+a) \cdot k+(j+b) \cdot d]$. Moreover, because $C$ is an identifying code, we have $i \cdot k+j \cdot d \equiv(i+a) \cdot k+$ $(j+b) \cdot d(\bmod n)$. Hence, $a \cdot k+b \cdot d \equiv 0(\bmod n)$. However, $(a, b) \in K, I(\mathscr{K}, x) \neq I(\mathscr{K}, y)$ by verifying all cases of $(a, b)$ which contradicts to $I(\mathscr{K} ; x)=I(\mathscr{K} ; y)$, so $C_{\mathscr{K}}$ is an identifying code in $\mathscr{K}$.

The proof is similar for the locating codes. Just notice that the vertices $x=(i, j) \in \mathbb{Z}^{2}$ and $y=x+(a, b) \in \mathbb{Z}^{2}$ are non-codewords in $\mathscr{K}$, and their corresponding vertices $i$. $k+j \cdot d$ and $(i+a) \cdot k+(j+b) \cdot d$ are also non-codewords in $\Gamma$.

Now, we give the proof for self-identifying codes. Suppose that $C$ is a self-identifying code in $\Gamma$ (it is also an identifying code) and $C_{\mathscr{K}}$ is an identifying code in $\mathscr{K}$. Next, we show that $I(\mathscr{K} ; x), I(\mathscr{K} ; y) \neq \varnothing$ for any distinct vertices $x=(i, j) \in \mathbb{Z}^{2}$ and $y=x+(p, q) \in \mathbb{Z}^{2}(p, q \in \mathbb{Z})$. Since $C_{\mathscr{K}}$ is an identifying code, the result is true if $d_{\mathscr{K}}(x, y) \geq 3$.

Suppose that $1 \leq d_{\mathscr{K}}(x, y) \leq 2$, namely, $\quad(p, q) \in$ $\{(-2,2),(-2,1),(-2,0),(-2,-1),(-2,-2),(-1,-2),(0,-2)$, $(1,-2),(2,-2), \quad(2,-1),(2,0),(2,1),(2,2),(1,2),(0,2)$, $(-1,2), \quad(-1,-1), \quad(0,-1), \quad(1,-1),(1,0),(1,1),(0,1)$, $(-1,1),(-1,0)\}$. Hence, the set $I(\Gamma ; i \cdot k+j \cdot d), I(\Gamma ;(i+$ $p) \cdot k+(j+q) \cdot d)$ contains a codeword of $C$ because $C$ is a self-identifying code in $\Gamma$. Notice that $i \cdot k+j \cdot d$ and $(i+$ $p) \cdot k+(j+q) \cdot d$ are distinct vertices by verifying all of the above $(p, q)$ cases in $\Gamma$ as $n>2 d>4 k, 2 d+k \neq n$, and $2 d+2 k \neq n$. Thus, $I[\mathscr{K} ; x], I[\mathscr{K} ; y]$ always contains a codeword of $C_{\mathscr{K}}$. This completes the proof.

Proof. of Theorems 1, 2, and 3. According to Table 1 and Lemmas 2, 3, and 4, the three theorems hold. 
TABLE 1: The densities of optimal identifying, locating-dominating, and self-identifying in $\mathcal{S}, \mathscr{T}$, and $\mathscr{K}$.

\begin{tabular}{lccc}
\hline & Square grid $\mathcal{S}$ & Triangular grid $\mathscr{T}$ & King grid $\mathscr{K}$ \\
\hline LD & $3 / 10[13]$ & & $1 / 5[8]$ \\
ID & $7 / 20[14,15]$ & $1 / 4[4]$ & $2 / 9[16,17]$ \\
Self-ID & $1 / 2[18]$ & $1 / 2[18]$ & $1 / 3[18]$ \\
\hline
\end{tabular}

\section{The Constructions for Lower Bounds}

In this section, we will give codes in the circulant graphs of meeting or infinitely approaching the lower bounds. The following lemma gives optimal ID codes and LD codes in $C_{n}(k, d)$.

Theorem 4. Let $C_{n}(k, d)$ be a circulant graph with $k, d$, and $n$ being positive integers such that $n>2 d>2 k \geq 2$ and $(k, d, n)=1$.

(i) If $\quad n \equiv 0(\bmod 20), \quad k \equiv 2(\bmod 20), \quad$ and $d \equiv 3(\bmod 20)$, then $\gamma^{I D}\left(C_{n}(k, d)\right)=(7 n / 20)$.

(ii) If $\quad n \equiv 0(\bmod 20), \quad k \equiv 3(\bmod 20), \quad$ and $d \equiv 5(\bmod 20)$, then $\gamma^{L D}\left(C_{n}(k, d)\right)=(3 n / 10)$.

Proof

Case 1: let $n \equiv 0(\bmod 20), \quad k \equiv 2(\bmod 20)$, and $d \equiv 3(\bmod 20)$. We define $B_{1}=\{0,4,6,7,13,14,16\}$ and

$$
C_{1}=\left\{u \in \mathbb{Z}_{n} \mid u \equiv b(\bmod 20) \text { for } b \in B_{1}\right\} .
$$

It is easy to verify that $B_{1}$ is an identifying code in $C_{20}(2,3)$, where $(2,3,20)=1$. Next, if we prove that all the $I$-sets $I\left(C_{n}(k, d), C_{1} ; x\right)$ are nonempty and unique, then $C_{1}$ is an identifying code in $C_{n}(k, d)$. According to the structure of $C_{1}$, it is easy to know that

$$
I\left(C_{20}(2,3), B_{1} ; x_{0}\right) \equiv I\left(C_{n}(k, d), C_{1} ; x\right)(\bmod 20),
$$

where $x \equiv x_{0}(\bmod 20)$ is an integer and $0 \leq x_{0} \leq 19$. Hence, $I\left(C_{n}(k, d), C_{1} ; x\right)$ are nonempty for all $x \in \mathbb{Z}_{n}$. Next, suppose $x \in \mathbb{Z}_{n}$ and $y \in \mathbb{Z}_{n}$ are distinct vertices, and the following two cases are discussed:

Case 1.1: $x \neq y(\bmod 20)$ : let $x^{\prime}$ and $y^{\prime}$ be integers such that $x=x^{\prime}(\bmod 20), y=y^{\prime}(\bmod 20), 0 \leq x^{\prime} \leq 19$, and $0 \leq y^{\prime} \leq 19$. Hence, if $I\left(C_{n} \quad(k, d), C_{1} ; x\right)$ $=I\left(C_{n}(k, d), \quad C_{1} ; y\right), \quad$ then $I\left(C_{20}(2,3), B_{1} ; x^{\prime}\right)=I\left(C_{20}(2,3), B_{1} ; y^{\prime}\right)$. This contradicts the fact that $B_{1}$ is the identifying code in $\mathrm{C}_{20}(2,3)$.

Case 1.2: $x \equiv y(\bmod 20):$ Next, we show that $N\left[C_{n}(k, d), C_{1} ; x\right] \cap N\left[C_{n}(k, d), C_{1} ; y\right]=\varnothing$.

Assume that there are $x, y \in Z_{n}$ such that $x+h=y+$ $h^{\prime} \in N\left[C_{n}(k, d), C_{1} ; x\right] \cap N\left[C_{n}(k, d), C_{1} ; y\right]$ for some $h, h^{\prime} \in\{-d,-k, 0, k, d\}$. We have $y+h^{\prime}-x-h=$ $0(\bmod 20)$, and then $h=h^{\prime}(\bmod 20)$ due to $x \equiv y(\bmod 20)$. So, $h=h^{\prime}$ and $x=y \quad$ (recalled $n>2 d>2 k)$. It is a contradiction. So, any vertex of $\mathbb{Z}_{n}$ is dominated by a codeword of $C_{1}$ and $I\left(C_{n}(k, d), C_{1} ; x\right) \neq I\left(C_{n}(k, d), C_{1} ; y\right)$. Thus, $C_{1}$ is an identifying code in $C_{n}(k, d)$.

Case 2: let $n \equiv 0(\bmod 20), \quad k \equiv 3(\bmod 20)$, and $d \equiv 5(\bmod 20)$. We define $B_{2}=\{0,1,2,11,12,13\}$ and

$$
C_{2}=\left\{u \in \mathbb{Z}_{n} \mid u \equiv b(\bmod 20) \text { for } b \in B_{2}\right\} .
$$

It is easy to verify that $B_{2}$ is an identifying code in $C_{20}(3,5)$, where $(3,5,20)=1$. Then, it is similar as in case 1 that $C_{2}$ is an identifying code in $C_{n}(k, d)$, where $(k, d, n)=1$ (just notice $x$ and $y$ are assumed to be non-codewords).

In the next theorem, we give an infinite sequence of identifying codes which infinitely approach the lower bound for identifying codes in Theorem 2 .

Theorem 5. Let $C_{n}(k, d-k, d)$ be a circulant graph with $k, d, n$ positive integers and $n>2 d>4 k,(k, d, n)=1$. Then, there is an infinite sequence of identifying codes in the circulant graphs $C_{n}(k, d-k, d)$ such that their density tends to $1 / 4$.

Proof. Let $k>1$ be even, $d=3 k-1$ be odd, and $n=6 k$. Let $S=\{j \mid 0 \leq j \leq k, j \equiv 0(\bmod 2)\}$. Define

$$
C_{d}=\left\{v \in \mathbb{Z}_{n} \mid v \equiv b(\bmod 2 k) \text { for some } b \in S\right\} .
$$

It is easy to see that code $C_{d}$ has order $3[k / 2+1]$. Therefore, $\lim _{k \rightarrow \infty}\left|C_{d}\right| / n=1 / 4$.

Next, we will prove that $C_{d}$ is an identifying code in $C_{n}(k, d-k, d)$.

Case 1: if $k \leq s \leq 2 k-1$ and $x \equiv s(\bmod 2 k)$ is odd, we can obtain $\{x-d, x+d\} \subseteq I(x)$. Because $N[x+d]$ $\cap N[x-d]=\{x\}$, we know only $I(x)$ contains elements $x+d$ and $x-d$ at the same time. So, $I(x) \neq I(y)$ for any $x \neq y$. If $k \leq s \leq 2 k-1$ or $s=0$ and $x \equiv s(\bmod 2 k) \quad$ is even, then we have $\{x-k, x+k\} \subseteq I(x)$. Since $N[x+k] \cap N[x-k]=\{x\}$, $I(x) \neq I(y)$ for any $x \neq y$.

Case 2: if $1 \leq s \leq k-1$ and $x \equiv s(\bmod 2 k)$ is odd, we know $\{x-d+k, x+d-k\} \subseteq I(x)$. Since $N[x-d+k]$ $\cap N[x+d-k]=\{x\}$, we know only $I(x)$ contains elements $x+d-k$ and $x-d+k$ at the same time. So, $I(x) \neq I(y)$ for any $x \neq y$. If $1 \leq s \leq k-1$ and $x \equiv s(\bmod 2 k)$ is even, then we have $I(x)=\{x\}$.

In summary, $C_{d}$ is an identifying code in $C_{n}(k, d-k, d)$ and their density tends to $1 / 4$.

Now, we give the construction of optimal locating and identifying codes in the circulant graph $C_{n}(k, d-k, d, d+k)$.

Theorem 6. Let $C_{n}(k, d-k, d, d+k)$ be a circulant graph with $k, d, n$ positive integers such that $n>2 d>4 k$, $2 d+k \neq n, 2 d+2 k \neq n$, and $(k, d, n)=1$. 
(i) For $k=2, d=9, n \geq 30$, and $n \equiv 0(\bmod 10)$, then $\gamma^{L D}\left(C_{n}(2,7,9,11)\right)=(n / 5)$.

(ii) We have an infinite sequence of identifying codes in the circulant graphs $C_{n}(k, d-k, d, d+k)$ such that their density tends to $2 / 9$.

Proof.

(i) Let $k=2, d=9, n \geq 30$, and $n \equiv 0(\bmod 10)$. Denote a code

$C^{\prime}=\left\{v \in \mathbb{Z}_{n} \mid v \equiv 0(\bmod 10)\right.$ or $\left.v \equiv 6(\bmod 10)\right\}$.

Next, we will verify $C^{\prime}$ is a locating code in $C_{n}(2,7,9,11)$.

According to the above conditions, we can obtain the $I$-sets of non-codewords $x$ of modulo 10 .

Let $x \neq y$. Next, we just need prove that $I(x) \neq I(y)$ for any non-codewords $x$ and $y$, and the following two cases are discussed.

Case 1: $x \neq y(\bmod 10)$ : according to Table 2 , it is easy to see that $I(x) \neq I(y)$ except $x \equiv 3(\bmod 10)$ and $y \equiv 8(\bmod 10)$. Therefore, we consider this special case. We know that $I(x)=\{x-7, x+7\}$ and $I(y)=\{y-2, y+2\}$. If $I(x)=I(y)$, owing to the data in columns $I(x)$ and $I(x)(\bmod 10)$, we have

$$
\left\{\begin{array}{l}
{[c] x-7 \equiv y-2(\bmod n)} \\
x+7 \equiv y+2(\bmod n)
\end{array}\right.
$$

It implies that, for any $0 \leq x, y \leq n-1$,

$$
\left\{\begin{array}{l}
2 x \equiv 2 y(\bmod n) \\
|y-x|=5
\end{array}\right.
$$

Assume that $y>x$ (otherwise, exchange the roles of $x$ and $y$ ), then

$$
\left\{\begin{array}{l}
2 x \equiv 2 y(\bmod n) \\
y-x=5
\end{array}\right.
$$

So, $2 x \neq 2 x+10(\bmod n)$ when $n \geq 30$ (only $n=10$ equals sign holds). Thus, $2 x \neq 2 y(\bmod n)$ if $n \geq 30$ and $n \equiv 0(\bmod n)$ and $I(x) \neq I(y)$ for any $x \neq y(\bmod 10)$.

Case 2: $x \equiv y(\bmod 10) .:$ in this case, the codewords in $I(x)$ are translated along $\mathbb{Z}_{n}$ to obtain the codewords in $I(y)$, so $I(x) \neq I(y)$ for any $x \equiv y(\bmod 10)$.

(ii) Let $k=5, d \geq 15, d \equiv 3(\bmod 6)$, and $n=3 d-9$ $(n \equiv 0(\bmod 6))$. Next, we divide the vertices of the circulant graph into three parts: $A_{1}=\{0,1,2, \ldots, d-1\}$, $A_{2}=\{d, d+1, d+2, \ldots, 2 d-1\}$, and $A_{3}=\{0,1,2, \ldots, n-1\},\left(A_{1} \cup A_{2}\right)$. We define the code
TABLE 2: $I(x)$ and $I(x)(\bmod 10)$.

\begin{tabular}{lcc}
\hline$x(\bmod 10)$ & $I(x)$ & $I(x)(\bmod 10)$ \\
\hline 1 & $\{x-11, x+9\}$ & 0,0 \\
2 & $\{x-2\}$ & 0 \\
3 & $\{x-7, x+7\}$ & 6,0 \\
4 & $\{x+2\}$ & 6 \\
5 & $\{x-9, x+11\}$ & 6,6 \\
7 & $\{x-7, x-11, x+9\}$ & $0,6,6$ \\
8 & $\{x-2, x+2\}$ & 6,0 \\
9 & $\{x-9, x+11, x+7\}$ & $0,0,6$. \\
\hline
\end{tabular}

$$
\begin{aligned}
C_{d}= & \left\{v \mid v \in\left(A_{1} \cup A_{3}\right), v \equiv 5(\bmod 6)\right\} \\
& \cup\left\{v \mid v \in A_{2}, v \equiv 0,4(\bmod 6)\right\} .
\end{aligned}
$$

Next, we will add another 7 codewords to $C_{d}$ and get $C_{d}^{\prime}$, so as to construct an identifying code of circulant graph. The value $C_{d} / n$ tends to $2 / 9$ when $d$ tends to infinity. Firstly, we remove some "borderline" vertices from above three parts and denote $A_{1}^{\prime}=A_{1}$, $\{0,1,2,3,4,5,6,7,8,9,10,11,12,13, d-5, d-4, d-3, d-2$, $d-1\}, \quad A_{2}^{\prime}=A_{2},\{d, d+1, d+2, d+3, d+4,2 d-5,2 d-$ $4,2 d-3,2 d-2,2 d-1\}$, and $A_{3}^{\prime}=A_{3},\{2 d, 2 d+1,2 d+$ $2,2 d+3,2 d+4,3 d-14,3 d-13,3 d-12,3 d-11,3$ $d-10\}$. These borderline vertices are discussed at the end. Let us first check the $I$-sets $I(x)$ for $x \in A_{1}^{\prime} \cup A_{2}^{\prime} \cup A_{3}^{\prime}$ (notice that $I(x)$ is obtained by relying on $C_{d}$ ).

According to Tables $3-5$, these $I$-sets are analyzed and compared. Obviously, when $|I(x)|=1$, the $I$-sets are different. Now, consider the case of $|I(x)|=2$. In Tables 3, 4, and 5 , we can see the distances $d\left(c_{1}, c_{2}\right)=c_{1}-c_{2}$ with $c_{1}>c_{2}$. If the distance $d\left(c_{1}, c_{2}\right)$ is different, the $I$-sets are also different, and if the distance $d\left(c_{1}, c_{2}\right)$ is same, the $c_{1}$ (mod6) and $c_{2}$ (mod6) are different as shown in Tables $3-5$, so the $I$-sets cannot be the same. The following is the case of $|I(x)|=3$. From Tables 3,4 , and 5 , we can see that $I$-sets are different except for $x \in A_{1}^{\prime}$, where $x \equiv 2(\bmod 6)$ and $y \in A_{3}^{\prime}$ $(y \equiv 2(\bmod 6))$. However, through verification, we can get $d\left(c_{1}, c_{2}\right)=d-4$ in $I(x)$, where $c_{1}=5(\bmod 6)$, $c_{2}=0(\bmod 6)$, and $d\left(c_{1}, c_{2}\right)=d-14$ in $I(y)$, where $c_{1}=5(\bmod 6)$ and $c_{2}=0(\bmod 6)$. Consequently, $I(x) \neq I(y)$ if $x, y \in A_{1}^{\prime} \cup A_{2}^{\prime} \cup A_{3}^{\prime}$ and $x \neq y$.

For the borderline vertices, we have the following $I$-sets distinguished: $I(0)=\{5,2 d-14\}, I(1)=\{d-4, d+1,2 d$ $-8\}, I(2)=\{d+7,2 d-12,2 d-2\}, I(3)=\{d+3,2 d-6\}$, $I(5)=\{5\}, \quad I(6)=\{11, d+1,2 d-8\}, \quad I(7)=\{d+7,2$ $d-2\}, \quad I(8)=\{d+3, d+13,2 d-6\}, \quad I(9)=\{d+9,2 d$ $+5\}, \quad I(10)=\{5, d+15\}, \quad I(11)=\{11\}, \quad I(12)=\{17, d+$ $7,2 d-2\}, \quad I(d-2)=\{d+3,2 d-2,3 d-16\}, \quad I(2$ $d-3)=\{11,2 d-8\}, \quad I(2 d+3)=\{17, d+3,2 d-2\}$, $I(3 d-14)=\{d-10,2 d-14\}, \quad I(3 d-13)=\{d-4, d+$ $1,2 d-18,2 d-8\}, I(3 d-11)=\{d+3,2 d-6,3 d-16\}$, and $I(3 d-10)=\{3 d-10\}$. We verify the $I$-sets of remaining vertices by considering $I(x), I(x)(\bmod 6)$, and the distance between codewords: $I(4)=\{d+9,3$ $d-10\}=I(d+4), \quad I(13)=\{d+13\}=I(d+13)$, $I(d-5)=\{d-10\}=I(d-10), I(d-4)=\{d-4, d+1\}=$ 
TABle 3: $I(x)$ and $I(x)(\bmod 10)$.

\begin{tabular}{lccc}
\hline$x \in A_{1}^{\prime}$ & $\mathrm{I}(x)$ & $d\left(c_{1}, c_{2}\right)$ & $I(x)(\bmod 6)$ \\
\hline $0(\bmod 6)$ & $\{x+5, x+d-5\}$ & $d-10$ & 5,4 \\
1 & $\{x-d-5, x+d\}$ & $d-14$ & 5,4 \\
2 & $\{x-d, x+d-5, x+d+5\}$ & & $5,0,4$ \\
3 & $\{x-d+5, x+d\}$ & $d-4$ & 5,0 \\
4 & $\{x-5, x+d+5\}$ & $d+10$ & 5,0 \\
5 & $\{x\}$ & & 5 \\
\hline
\end{tabular}

TABLE 4: $I(x)$ and $I(x)(\bmod 10)$.

\begin{tabular}{lccc}
\hline$x \in A_{2}^{\prime}$ & $I(x)$ & $d\left(c_{1}, c_{2}\right)$ & $I(x)(\bmod 6)$ \\
\hline $0(\bmod 6)$ & $\{x\}$ & & 0 \\
1 & $\{x-d-5, x+5, x+d-5\}$ & & $5,0,5$ \\
2 & $\{x-d, x+d\}$ & $d-9$ & 5,5 \\
3 & $\{x-d+5, x-5, x+d+5\}$ & & $5,4,5$ \\
4 & $\{x\}$ & & 4 \\
5 & $\{x-5, x+5\}$ & 10 & 0,4 \\
\hline
\end{tabular}

TABLE 5: $I(x)$ and $I(x)(\bmod 10)$.

\begin{tabular}{lccc}
\hline$x \in A_{3}^{\prime}$ & $I(x)$ & $d\left(c_{1}, c_{2}\right)$ & $I(x)(\bmod 6)$ \\
\hline 0 (mod6) & $\{x-d-5, x+5\}$ & $d+10$ & 4,5 \\
1 & $\{x-d, x+d-5\}$ & $d-4$ & 4,5 \\
2 & $\{x-d-5, x-d+5, x+d\}$ & & $0,4,5$ \\
3 & $\{x-d, x+d+5\}$ & $d-14$ & 0,5 \\
4 & $\{x-d+5, x-5\}$ & $d-10$ & 0,5 \\
5 & $\{x\}$ & & 5 \\
\hline
\end{tabular}

$I(d+1), \quad I(d-3)=\{2 d-8\}=I(2 d-8), \quad I(d-1)=$ $\{2 d-6,3 d-10\}=I(d-6), I(d)=\{5,2 d+5\}=I(d+5)$, $I(d+2)=\{d+7\}=I(d+7), I(d+3)=\{d+3,2 d-2\}=$ $I(2 d-2), \quad I(2 d-5)=\{d-10,3 d-10\}=I(2 d-10)$, $I(2 d-4)=\{5, d-4, d+1\}=I(2 d+1), \quad I(2 d-1)=$ $\{2 d-6\}=I(2 d-6), \quad I(2 d)=\{2 d+5\}=I(2 d+5), \quad I$ $(2 d+2)=\{11, d+7\}=I(2 d+7), I(2 d+4)=\{d+9\}=$ $I(d+9)$, and $I(3 d-12)=\{2 d-12\}=I(2 d-12)$. We add seven more codewords, namely, $D=\{0,6,9,12, d$ $-7,2 d+4,2 d+10\}$ to the code $C_{d}$ to avoid these same $I$-sets. The new code is denoted by $C_{d}^{\prime}=C_{d} \cup D$. Now let us verify $I(x)$ based on $C_{d}^{\prime}$. We know that if $I\left(C_{d}, x\right) \neq I\left(C_{d}, y\right)$, then $I\left(C_{d}^{\prime}, x\right) \neq I\left(C_{d}^{\prime}, y\right)$. In addition, $0 \in I(d-5), I(d-10)$, $0 \in I(2 d-4), I(2 d+1), 6 \in I(2 d-8), I(d-3), 6 \in I(d$ $+1), I(d-4), 6 \in I(2 d+2), I(2 d+7), 9 \in I(d+9), I$ $(2 d+4), 9 \in I(2 d-5), I(2 d-10), 12 \in I(d+7), I(d$ $+2), \quad d-7 \in \quad I(2 d-12), I \quad(3 d-12), \quad d-7 \in I(2 d$ $-2), I(d+3), 2 d+4 \in I(2 d-1), I(2 d-6), 2 d+4 \in I$ (13), $I(d+13), \quad 2 d+4 \in I(d+4), I(4), 2 d+4 \in I(d$ -1), $I(d-6), 2 d+10 \in I(2 d+5), I(2 d)$, and $2 d+10$ $\in I(d+5), I(d)$. Therefore, $C_{d}^{\prime}$ is an identifying code and its density tends to $2 / 9$.

In the following theorem, we will show that there are infinite families of circulants in which the lower bounds of self-identifying codes in Theorem 1, 2, and 3 can be reached.
Theorem 7. Let $k, d$, and $n$ be integers such that $d \geq 4$ $(k, d, n)=1$.

(i) If $k$ is odd, $d$ is even, $d>k, n \geq 4 d+1$, and $n \equiv 0(\bmod 2)$, then $\gamma^{S I D}\left(C_{n}(k, d)\right)=(n / 2)$.

(ii) If $k$ is odd, $d>k, n \geq 4 d+1$, and $n \equiv 0(\bmod 2)$, then $\gamma^{S I D}\left(C_{n}(k, d-k, d)\right)=(n / 2)$.

(iii) If $k \equiv 1(\bmod 3), \quad d \equiv 1(\bmod 3), \quad d>k$, $n \geq 4 d+4 k+1, \quad$ and $n \equiv 0(\bmod 3)$, then $\gamma^{S I D}\left(C_{n}(k, d-k, d, d+k)\right)=(n / 3)$.

Proof

(i) Let the code $C=\left\{v \in \mathbb{Z}_{n} \mid v \equiv 0(\bmod 2)\right\}$, $|C|=(n / 2)$. We will show that $C$ is a self-identifying code in $C_{n}(k, d)$. If $x \equiv 0(\bmod 2)$, then $I(x)=\{x-d, x, x+d\}$. If $x \equiv 1(\bmod 2)$, then $I(x)=\{x-k, x+k\}$. Because $n \geq 4 d+1$, we have $N[x-d] \cap N[x+d]=\{x\}$ and $N[x-k] \cap N$ $[x+k]=\{x\}$. This meets the definition of a selfidentifying code, which is $I(x) \neq \varnothing$ and $\cap_{c \in I(C ; x)} N[c]=\{x\}$. Thus, $C$ is a self-identifying code in $C_{n}(k, d)$ and $\gamma^{\operatorname{SID}}\left(C_{n}(k, d)\right)=(n / 2)$.

(ii) Let the code $C=\left\{v \in \mathbb{Z}_{n} \mid v \equiv 0(\bmod 2)\right\}$, $|C|=(n / 2)$. We will prove that $C$ is a self-identifying code in $C_{n}(k, d-k, d)$. Suppose $d$ is even (resp. odd); then, if $x \equiv 0(\bmod 2)$, there is $\{x-d, x+d\} \subseteq I(x) \quad$ (resp. $\quad\{x-d+k, x+d-k\}$ $\subseteq I(x))$, so $\cap_{c \in I(x)} N[c]=\{x\}$, and if $x \equiv 1(\bmod 2)$, there is $\{x-d+k, x+d-k\} \subseteq I(x) \quad$ (resp. $\{x-d, x+d\} \subseteq I(x)) ;$ hence, again $\cap_{c \in I(x)} N[c]$ $=\{x\}$. Therefore, $C$ is a self-identifying code in $C_{n}(k, d-k, d)$ and $\gamma^{S I D}\left(C_{n}(k, d-k, d)\right)=(n / 2)$.

(iii) Let $C=\left\{v \in \mathbb{Z}_{n} \mid v \equiv 0(\bmod 3)\right\},|C|=(n / 3)$. Next, we will prove that $C$ is a self-identifying code in $C_{n}(k, d-k, d, d+k)$. If $x \equiv 0(\bmod 3)$, we can get $I(x)=\{x-d+k, x, x+d-k\}$ due to $k=1(\bmod 3)$ and $d=1(\bmod 3)$. If $x \equiv 1(\bmod 3)$ (resp. $x \equiv 2(\bmod 3))$, then we have $I(x)=\{x-d$, $x-k, x+d+k\} \quad$ (resp. $I(x)=\{x-d-k, x+k$, $x+d\})$. Anyhow, the condition $\cap_{c \in I(x)} N[c]=\{x\}$ is satisfied due to the fact that $n \geq 4 d+4 k+1$. Thus, $C$ is a self-identifying code in $C_{n}(k, d-k, d, d+k)$ and $\gamma^{\operatorname{SID}}\left(C_{n}(k, d-k, d, d+k)\right)=(n / 3)$.

\section{Data Availability}

No data were used to support this study.

\section{Conflicts of Interest}

The authors declare that they have no conflicts of interest.

\section{Acknowledgments}

This work was partially supported by the NSFC (Nos. 61771019 and 11701497) and NSFS (No. AR2020MA044). 


\section{References}

[1] J. Ville and L. Tero, "Collection of codes for tolerant location," in Proceedings of the Bordeaux Graph Workshop, pp. 176-179, Bordeaux, France, 2016.

[2] J. Ville and L. Tero, "Tolerant location detection in sensor networks," Advances in Applied Mathematics, vol. 112, Article ID 101938, 2020.

[3] P. J. Slater, "Dominating and reference sets in a graph," Journal of Mathematical and Physics, vol. 22, no. 4, pp. $445-455,1988$.

[4] M. G. Karpovsky, K. Chakrabarty, and L. B. Levitin, "On a new class of codes for identifying vertices in graphs," IEEE Transactions on Information Theory, vol. 44, no. 2, pp. 599611, 1998.

[5] D. W. Cranston and G. Yu, "A new lower bound on the density of vertex identifying codes for the infinite hexagonal grid," Electronic Journal of Combinatorics, vol. 16, no. 1, pp. 1632-1655, 2010.

[6] G. Exoo, V. Junnila, T. Laihonen, and S. Ranto, "Improved bounds on identifying codes in binary hamming spaces," European Journal of Combinatorics, vol. 31, no. 3, pp. 813-827, 2010.

[7] T. W. Haynes, M. A. Henning, and J. Howard, "Locating and total dominating sets in trees," Discrete Applied Mathematics, vol. 154, no. 8, pp. 1293-1300, 2006.

[8] I. Honkala and T. Laihonen, "On locating-dominating sets in infinite grids," European Journal of Combinatorics, vol. 27, no. 2, pp. 218-227, 2006.

[9] T. Laihonen, "On cages admitting identifying codes," European Journal of Combinatorics, vol. 29, no. 3, pp. 737-741, 2008.

[10] T. Müller and J. S. Sereni, "Identifying and locating-dominating codes in (random) geometric networks," Combinatorics, Probability and Computing, vol. 18, no. 6, pp. 925-952, 2009.

[11] M. Ghebleh and L. Niepel, "Locating and identifying codes in circulant networks," Discrete Applied Mathematics, vol. 161, no. 13-14, pp. 2001-2007, 2013.

[12] J. Ville, L. Tero, and P. Gabrielle, "Optimal bounds on codes for location in circulant graphs," Cryptography and Communications, vol. 11, pp. 621-640, 2019.

[13] P. J. Slater, "Fault-tolerant locating-dominating sets," Discrete Mathematics, vol. 249, no. 1-3, pp. 179-189, 2002.

[14] Y. Ben-Haim and S. Litsyn, "Exact minimum density of codes identifying vertices in the square grid," SIAM Journal on Discrete Mathematics, vol. 19, no. 1, pp. 69-82, 2005.

[15] G. Cohen, S. Gravier, I. Honkala et al., "Improved identifying codes for the grid," Electronic Journal of Combinatorics, vol. 6 , 1999.

[16] I. Charon, O. Hudry, and A. Lobstein, "Identifying codes with small radius in some infinite regular graphs," The Electronic Journal of Combinatorics, vol. 9, no. 1, 2002.

[17] G. D. Cohen, L. Honkala, A. Lobstein, and G. Zémor, "On codes identifying vertices in the two-dimensional square lattice with diagonals," IEEE Transactions on Computers, vol. 50, no. 2, pp. 174-176, 2001.

[18] I. Honkala and T. Laihonen, "On a new class of identifying codes in graphs," Information Processing Letters, vol. 102, no. 2-3, pp. 92-98, 2007. 